\title{
Initial Psychological Responses to Swine Flu
}

\author{
Robin Goodwin • Stanley O. Gaines Jr • Lynn Myers • \\ Felix Neto
}

Published online: 2 March 2010

(C) International Society of Behavioral Medicine 2010

\begin{abstract}
Background The emergence of influenza A ("swine flu") in early 2009 led to widespread public concern. However, little research has examined the factors that underlie initial worry about infection and subsequent behavioral responses to such worry.

Purpose This study seeks to model some key predictors of worry and behavioral responses in the early stages of the swine flu pandemic (WHO pandemic stage 5).

Method A cross-sectional internet questionnaire study $(N=186)$.

Results Twenty-five percent of respondents rated themselves as worried about being a victim of swine flu, $40 \%$ that they were worried of a family member contracting the virus. Twenty percent had bought, or intended to buy, preparatory materials (e.g., face masks), $20 \%$ intended to delay or cancel air travel. In a structural equation model, conservation values and family or friends perception of risks predicted worry about infection, while worry correlated with the purchase of preparatory materials, a lesser willingness to travel by public transport, and difficulty in focusing on everyday activities.

Conclusion While previous research on pandemic risk perception has focused on cognitive risk judgments, our
\end{abstract}

Author's contributions RG, FN, and LM designed and conceived the overall project. SG analyzed the data along with RG. LM, RG, and SG helped in data interpretation and FN in drafting the final article.

R. Goodwin $(\bowtie) \cdot$ S. O. Gaines Jr · L. Myers

School of Social Sciences, Brunel University,

Uxbridge UB8 3PH, UK

e-mail: robin.goodwin@brunel.ac.uk

F. Neto

Faculdade de Psicologia e de Ciencias da Educacao,

Universidade do Porto,

Porto, Portugal data suggests that initial "emotional" concerns about infection are also significant predictors of behavioral responses to pandemic threat. Such worry is likely to be influenced by a variety of individual factors, such as personal values, as well as normative pressures. Practitioners can use and expand on such models of pandemic response when tailoring health campaigns to meet newly emergent threats.

Keywords Pandemic influenza · Worry Intrusive thoughts · Values

\section{Introduction}

Swine flu (H1N1) is a rapidly spreading influenza A virus transmitted between humans through coughing or sneezing or via contaminated hands or surfaces [1]. An outbreak of the virus in the spring of 2009 led to early fatalities in Mexico but rapidly proliferated worldwide. The WHO declared swine flu a global pandemic on 11 June 2009, by which time an estimated 24,000 had been infected, and 143 had died [1].

Despite great medical interest in pandemic influenza, there have been relatively few attempts to examine the factors underlying worry about infection and subsequent behavioral responses. Research on H5N1 transmission ("bird flu": [2]) and on psychological responses to SARS [3] found risk perceptions of infection to be a predictor of a range of preventive behaviors. In one of the few studies of swine flu, Cummings et al. examined determinants of immunization behavior in the 1976 swine flu influenza outbreak in the USA [4]. They found perceived susceptibility and severity of swine flu, alongside the perceived barriers to, and benefits of, action, were all significant determinants of vaccination. 
Most of these previous studies on psychological responses to influenza pandemics have, however, viewed risk primarily in cognitive terms, paying little attention to the more emotional correlates of health-related behaviors [5]. During a rapidly spreading influenza epidemic, when there is often limited evidence-based information, emotion may become of greater importance [5], and in our study we focus on the "emotional" dimension of worry about contracting swine flu. We define worry as an affective, emotional response to threat, and an important predictor of protective behaviors independent of severity of risk and risk likelihood estimates [6].Several factors are likely to influence worry about influenzas and subsequent behaviors, and new combined models may be required to analyze emergent threats [6]. We adapted an analytic framework derived from recent work response to terrorist threat [7]. This model suggests that demographic factors, social context factors (such as group norms), and individual values are all predictors of worry about a threat. This worry then predicts behaviors that address that threat, as well as the presence of intrusives that may interfere with daily activities. Following the SARS outbreak, women were more concerned than men about themselves or their family contracting the virus [8], and we also expect this sex difference in worry. Consistent with other research on avian influenza [9], we anticipate that older respondents will be less worried about swine flu. Worry about threat does not occur in a "vacuum," and individuals may rapidly "catch" the emotional reactions of others [10]. As a result, the perception that significant others are worried is likely to positively correlate with personal concern $[5,11]$. In line with previous work on external threat, we anticipate that those who hold "conservation" values (security, conformity, and tradition) are more likely to be concerned about threats to their personal and family safety [7]. Worry has been seen as a positive correlate of precautionary activities following a swine flu outbreak [12], and we also predict such an association. In addition, worry about a future threat has been significantly correlated with difficulty in focusing on everyday life and work activities [13] and we anticipate a similar association.

Concerns about infection from newly emergent diseases may change rapidly $[3,14]$, and one persisting problem when assessing worry and behavior is that of directionality: individuals may be motivated by their worry about particular behavior (e.g., vaccination) but this behavioral action (vaccination) may then reduce worry. This can then lead to apparently conflicting worry-behavior correlations [5, 15]. Data were collected early in the pandemic (WHO phase 5, prior to the announcement of the stage 6 , global pandemic). These initial responses should allow an examination of worry about infection relatively unmuddied by estimates influenced by the prospect of vaccination [15] and before continued exposure induces familiarity and habituation [9].

\section{Method}

\section{Participants}

Following ethics approval, 186 respondents completed an online questionnaire in English or Portuguese, linked to a website established for these purposes (age range 19-69, $M$ age 33.77 , SD 12.66, 68\% female). The website link was pasted onto a variety of general, non-health-related networking websites (e.g., "I love London") and also advertised through the groups' pages of social networking sites and announced during student classes. All but eleven respondents (94\%) were resident in Europe, with participants primarily of Portuguese $(N=80)$, British $(N=58)$, or Finnish $(N=21)$ origin and resident in Portugal (80 respondents), Britain (75 respondents), or Finland (22 respondents). Sixty-three percent of our respondents were students or had a college degree or above, $37 \%$ had only matriculated or had lesser education. Responses were gathered between April 29, 2009 and June 11, 2009; i.e., during WHO pandemic stage 5.

\section{Measures}

The questionnaire included demographic factors (gender, age), personal worldviews, normative beliefs about infection, worry about risk to friends and family, the purchasing of preparatory materials, changes in transport use, and intrusive thoughts that disrupted daily life. Values [16] were assessed using Schwartz' short values questionnaire [17]. The three conservation items (security, conformity, and tradition) were combined to form a single conservation score $(\alpha=84)$. Participants indicated the importance of each value on a 9item scale, ranging from opposed to supreme importance. Normative concerns about risk were measured by asking two questions: "How great a risk does your family (friends) think this pandemic is," ranging on a 4-point from very high to very low (plus "don't know"). These items were highly correlated $(0.69, p<0.001)$, and a single mean score was used for analysis. Personal worry was assessed with two questions: "How concerned are you personally about you, yourself (a member of your family) being the victim of this outbreak?" measured on a 4-point scale (from not at all concerned to very concerned). As these two items also correlated highly $(r=0.62, p<0.001)$, a single combined "worry" measure was used in the analysis.

Outcome behaviors were chosen as ones widely reported within the literature $[5,7]$. Outcome measures were (1) "have you bought, or do you intend to buy, anything in preparation for a swine flu epidemic?" (yes/no); 2) "As a result of the threat will use public transport..." less often (the same, more often); (3) "Will you cancel or delay any specific plans to travel by air now?" (yes/no); and (4) "Since the threat of swine flu, how difficult has it been, if at all, for you to 
concentrate on your job or your normal activities because of the way you feel about the threat?" (4-point scale, from very difficult to not at all difficult).

\section{Results}

Thirty-four percent of participants' family members and $35 \%$ of their friends were judged as rating the risk of swine flu to be "very high" or "moderately high" (NORM in the structural model, below). Twenty-five percent of participants rated themselves as "very or somewhat" worried about becoming a victim of swine flu, and $40 \%$ expressed they were "very/somewhat concerned" about a family member contracting the virus (WORRY). Twenty percent had bought or intended to buy materials in preparation for a wider pandemic (PREPARE); $20 \%$ intended to delay or cancel air travel plans (AIR), 22\% anticipated using public transport less frequently (TRANSPORT). Only 12\%, however, claimed that it had been "very difficult" or "somewhat/a little difficult" to focus on everyday life and work because of their worry about the threat (THOUGHT). There were no sex or age differences in the purchase of preparatory materials, the cancelation of air travel or reduction in public transport, or distracting anxieties about the threat (for sex differences, ts (184) -0.29 to -1.36 , ps $>$ 0.17 ; for age effects rs $(183)<0.10$, ps $>0.18)$

\section{Structural Model}

Structural equation modeling permits the examination of mediating variables and allows for the testing of models with multiple dependent variables. Prior to conducting structural equation analyses, we screened the data for multivariate normality via PRELIS [18]. Results of Mardia's test of relative multivariate kurtosis yielded a value of 1.25 , which is well below the cutoff point of 3.00 [19]. Thus, we concluded that the data were normal at the multivariate level. The matrix of correlations among the predictor, mediator, and criterion variables (available from the authors upon request) subsequently was entered into LISREL 8.72, using maximum likelihood estimation [20].

Results of an initial structural equation analysis indicated that the hypothesized model did not provide an adequate fit to the data $\left(\chi^{2}=63.98, \mathrm{df}=35, p<0.01\right.$; RMSEA $=0.07$; $\mathrm{CFI}=$ $0.85)$. However, inspection of residuals indicated that after controlling for two unexpected instances of correlated measurement error (i.e., between PREPARE and TRANSPORT and between AGE and CONSERVATION), results of a final structural equation analysis indicated that the hypothesized model did provide an adequate fit to the data $\left(\chi^{2}=33.61, \mathrm{df}=\right.$ 33 , NS; RMSEA $=0.00 ; \mathrm{CFI}=1.00$ ). Moreover, comparison of the initial and final structural equation analyses indicated that controlling for those instances of correlated measurement error resulted in a significant reduction in chi-square $\left(\chi^{2}\right.$ difference $=30.37, \mathrm{df}=2, p<0.01$ ).

$R^{2}$ values associated with the endogenous variables in the final model are as follows: 0.20 (WORRY), 0.18 (PREPARE), 0.12 (TRANSPORT), 0.15 (AIR), and 0.06 (THOUGHT). Power associated with prediction of the endogenous variables, calculated via GPower [21] was 1.00 for WORRY, 0.99 for PREPARE, 0.99 for TRANSPORT, 0.99 for AIR, and 0.86 for THOUGHT. Inspection of the beta coefficients in the structural portion of the model revealed that with the exception of the paths from AGE to WORRY and from SEX to WORRY, all of the paths were significant and in the expected direction $(\mathrm{ps}<0.01)$.

Taken together, these results indicate that those high on conservation values and those who estimate that their friends and family see the epidemic as posing a great risk demonstrate the most worry about themselves and their family's wellbeing. This concern then predicts a greater willingness to buy preparatory materials (such as face masks), more difficulty in concentrating on everyday work and activities because of worries about the epidemic, and a lesser willingness to travel by public transport or by airplane.

\section{Discussion}

The "Influenza A"/“swine flu" outbreak of early 2009 poses a potentially serious health challenge to large numbers of people around the world. In our preliminary study, we examined emotional concern about the pandemic and the consequences of such concern for behavior (Fig. 1). Respondents who held values emphasizing security, tradition, and conformity, and those who saw their friends and family as judging swine flu as a greater threat were the most worried about the pandemic. This worry correlated with preparatory and avoidance behaviors (buying materials such as face masks, reducing public transport use) and the presence of intrusive pandemic-related thoughts. There were no significant gender or age differences in threat perception or behavioral response.

Our data were collected in the early phases of the swine flu epidemic, during which time there had been relatively few deaths outside Mexico. Cultural experiences with pandemic threat are likely to modify pandemic responses [22] and it is therefore perhaps unsurprising that only a minority of our primarily European respondents reported high levels of personal concern, or had purchased, or intended to purchase, preparatory materials for the epidemic. Responses to pandemics are likely to be partly dependent on mortality rates [8], and may be highly time sensitive [3]. Worry at Time 1 can lead to later diminution of concern: coping mechanisms, such as the avoidance of crowds, can feed back into 


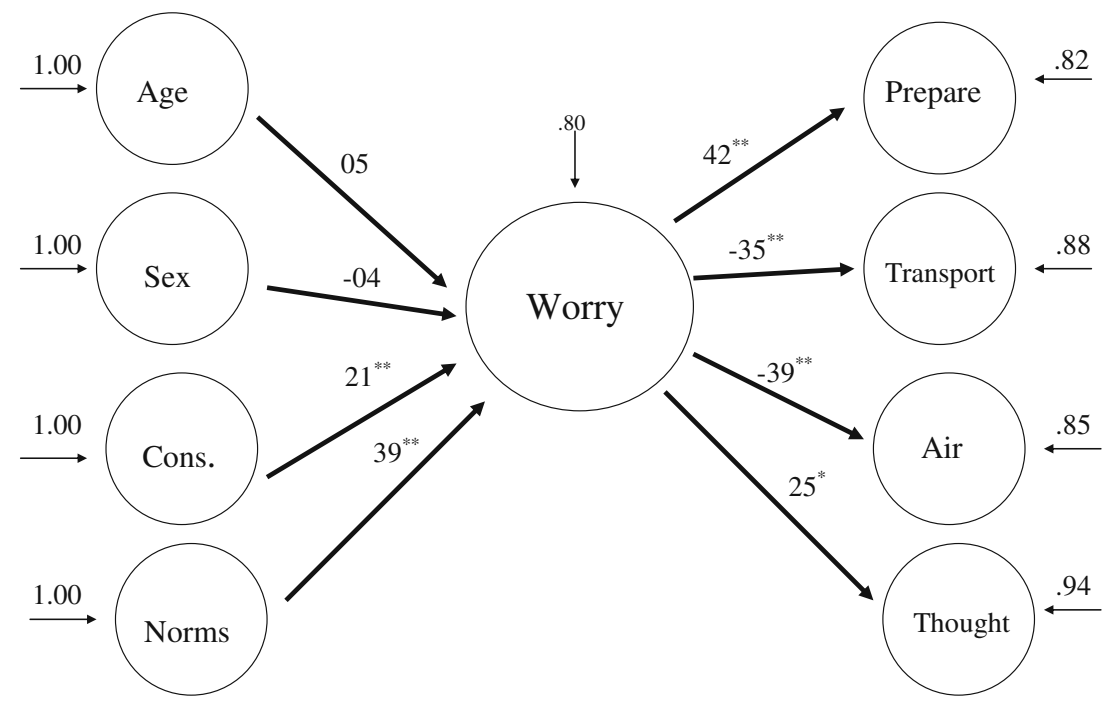

Fig. 1 Concern about swine flu and its consequences. $N=156 ; x^{2}=33.61, \mathrm{df}=33.0$; ns. Single asterisk indicates $p<.05$. Double asterisks indicate $p<0.01$

new, reduced worry [5]. Such reappraisals may be particularly significant if the coping is costly-for example, when avoiding crowds threatens an individual's travel to their workplace. Unfortunately, major influenza outbreaks often occur in waves, with a real risk that escaping infection in a first wave may lead to a misleading sense of immunity [5].

Our study has a number of theoretical and practical implications. Our findings demonstrate the strong correlation between perceptions of family and friends' anxieties and personal anxieties, underlining the potential risk of "emotional contagion" [10] between groups during a time of collective concern. This suggests a particular need to understand and address the functioning of social networks during times of pandemic threat. Recognizing the significance of individual value variations may also have a number of practical implications when attempting to motivate particular groups towards appropriate behaviors during a pandemic. Just as those who value conservation values may be sufficiently concerned to take particular actions, those with opposing values (in Schwartz's model [16], those who are high on self-direction, stimulation, and hedonism) may be less amenable to behavioral change. Indeed, work on the sexual behavior of such individuals suggests this latter group may have relatively little concern for their personal safety [23]. Health campaigners need to recognize this value diversity when attempting to promote particular behaviors during a pandemic and to modify their campaigns accordingly. Finally, our results emphasize the significance of emotional determinants of actions [6], particularly when there is considerable uncertainty about the magnitude of the threat [5]. Understanding these emotional responses has implications not only for healthrelated behaviors but for whole economies, with pandemics posing severe economic challenges to the tourism, transport, and leisure industries [14].

Our study had a number of limitations, and our results must be seen as clearly preliminary at this stage. This was an online study, and while this method is useful for rapid collection of data, and is likely to produce similar results to paper and pencil methods [24], ours was a small size convenience sample, with respondents selectively recruited. As such we did not use a true random sample, and the distribution of data in our sample cannot be seen as descriptive of different attitudes or behavioral responses in a general population. Such a voluntary sample may differ significantly from a general population on key personality variables, such as neuroticism, a concept which often includes indicators of concern and worry [25]. Although link between education and worry about infection from influenza viruses is uncertain [2], we also recognize the need for broader population sampling, particularly as our European sample was primarily from just three European countries - the UK, Portugal, and Finland. We measured normative concerns indirectlyassessing our respondent's perceptions of the risk estimates of their friends and family - and future work could directly measure such risk assessments from these friends and family. Our data were collected cross-sectionally, limiting our ability to examine causal relations between the variables in our model, with prospective studies needed to help unpack the relationship between worry and behavior [15]. As the pandemic develops, and as individuals have greater opportunities to consider the range of possible behavioral responses and the personal implications of these, other factors (e.g., the cost of avoiding particular areas, the perceived effectiveness of an action) should be included. Additional cognitive risk perceptions might mediate the 
relationship between emotional concern and behavioral response, and a full test of the relative contribution of emotional concerns requires testing its impact on behavior above and beyond the cognitive dimension of risk perception. Finally, the use of dichotomous outcome measures to assess relatively "rare" outcomes (e.g., the purchasing of goods) can lead to an underestimation of the clinical significance of concern as a predictor of behavior [26].

The emergence of a new pandemic influenza provides a major challenge to behavioral scientists, demanding consideration not only of the traditional cognitive estimates of risk but recognition too of the significant role of emotional worries in predicting behavioral outcomes. This emotional concern is itself likely to be influenced by several individual and group factors. Future work should attempt to replicate the current results with larger samples, further predictors and correlates of worry, and a greater range of demographic indicators (such as marital status and number of children). Such work should help facilitate intervention strategies that maximize the potential for the positive behavioral changes needed to confront such pandemic threats.

Acknowledgments We would like to thank Hazel George, Önver A. Cetrez, and Arniika Kuusisto for their help with data collection, and two non-anonymous reviewers for their helpful feedback on our original submission.

Competing interests All authors declare that they have no competing interests.

\section{References}

1. World Health Organization. Reducing excess mortality from common illnesses during an influenza pandemic. 2008. http://www.who. int/diseasecontrol_emergencies/common_illnesses2008_6.pdf. Accessed 20th June 2009.

2. Lau TFJ, Kim JH, Tsui HY, Griffiths S. Perceptions related to bird-to-human avian influenza, influenza vaccination, and use of face mask. Infection. 2008;36:434-43.

3. Blendon RJ, Benson JM, DesRoches CM, Raleigh E, TaylorClark K. The public's response so severe acute respiratory syndrome in Toronto and the United States. Clin Infect Dis. 2004;38:925-31.

4. Cummings KM, Jette AM, Brock BM, Haefner DP. Psychosocial determinants of immunization behaviour in a swine influenza campaign. Med Care. 1979;17:639-49.

5. Leppin A, Aro AR. Risk perceptions related to SARS and avian influenza: theoretical foundations of current empirical research. Int J Behav Med. 2009;16:7-29.
6. Schmiege SJ, Bryan A, Klein WMP. Distinctions between worry and perceived risk in the context of the theory of planned behavior. J Appl Social Psychology. 2009;39:95-119.

7. Goodwin R, Gaines S. Terrorism perception and its consequences following the 7th July 2005 London Bombings. Behav Sci Terrorism Political Aggression. 2009;1:50-65.

8. Lau TFJ, Kim JH, Tsui HY, Griffiths S. Anticipated and current preventive behaviors in response to an anticipated human-tohuman H5N1 epidemic in the Hong Kong Chinese general population. BMC Infect Dis. 2007;7:1-18.

9. Fielding R, Lam WW, Ho EY, Lam TH, Hedley AJ, Leung GM. Avian influenza risk perception, Hong Kong. Emerg Infect Dis. 2005; $11: 677-82$.

10. Hatfield E, Cacioppo J, Rapson RL. Emotional contagion. Cambridge: Cambridge University Press; 1994.

11. Pidgeon RE, Kasperson RE, Slovic P. The social amplification of risk. Cambridge: Cambridge University Press; 2003.

12. Rubin GJ, Amlôt R, Page L, Wessely S. Public perceptions, anxiety, and behavioural change in relation to the swine flu outbreak: cross sectional telephone survey. BMJ. 2009;339:b2651.

13. Sprang G. Post-disaster stress following the Oklahoma City bombing: an examination of three community groups. J Interpers Violence. 1999;14:161-75.

14. Smith RD. Responding to global infectious disease outbreaks: lessons from SARS on the role of risk perception, communication and management. Soc Sci Med. 2006;63:3113-23.

15. Brewer NT, Chapman GB, Gibbons FX, Gerard M, McCaul KD, Weinstein ND. A meta-analysis of the relationship between risk perception and health behavior: the example of vaccination. Health Psychol. 2007;26:136-45.

16. Schwartz SH. Universals in the content and structure of values: theory and empirical tests in 20 countries. In: Zanna M, editor. Advances in experimental social psychology, vol. 25. New York: Academic; 1992. p. 1-65.

17. Lindeman M, Verkasalo M. Measuring values with the short Schwartz's value survey. J Pers Assess. 2005;85:170-8.

18. Joreskog K, Sorbom D. PRELIS 2: user's reference guide. Chicago: Scientific Software international; 1996.

19. DeCarlo LT. On the meaning and use of kurtosis. Psychol Methods. 1997;2:292-307.

20. Joreskog K, Sorbom D. LISREL 8.72. Chicago: Scientific Software International; 2005.

21. Faul F. GPower 3.0.5 (Computer program). Kiel: Universitaat Kiel; 2009. http://www.psycho.uni-duesseldorf.de/aap/projects/ gpower Accessed 15th October.

22. DeZwart O, Veldhuijzen IK, Elam G, Aro AR, Abraham T, Bishop GD, et al. Avian Influenza, risk perception, Europe and Asia. Emerg Infect Dis. 2007;13:290-3.

23. Goodwin R, Realo A, Kwiatkowska A, Kozlova A, Nguyen-Luu LA, Nizharadze G. Values and sexual behavior in central and Eastern Europe. J Health Psychol. 2002;7:45-56.

24. Murray D, Fisher D. The internet: a virtually untapped tool for research. J Tech Human Services. 2002;19:5-18.

25. Lönnqvist J-E, Paunonen S, Verkasalo M, Leikas S, TuulioHenriksson A, Lönnqvist J. Personality characteristics of research volunteers. Eur J Pers. 2007;21:1017-30.

26. Rutledge T, Loh C. Effect sizes and statistical testing in the determination of clinical significance in behavioral medicine research. Ann Behav Med. 2004;272:134-45. 